Trinity College

Trinity College Digital Repository

Faculty Scholarship

2004

Chemistry-induced Intrinsic Stress Variations During the Chemical Vapor Deposition of Polycrystalline Diamond

\author{
Ashok Rajamani \\ Brown University \\ Brian W. Sheldon \\ Brown University \\ Sumit Nijhawan \\ Brown University \\ Alan Schwartzman \\ Brown University \\ Janet Rankin \\ Brown University
}

See next page for additional authors

Follow this and additional works at: https://digitalrepository.trincoll.edu/facpub

Part of the Physics Commons 


\section{Authors}

Ashok Rajamani, Brian W. Sheldon, Sumit Nijhawan, Alan Schwartzman, Janet Rankin, Barbara L. Walden, and Laura Riester 


\title{
Chemistry-induced intrinsic stress variations during the chemical vapor deposition of polycrystalline diamond
}

\author{
Ashok Rajamani, Brian W. Sheldon, Sumit Nijhawan, Alan Schwartzman, and \\ Janet Rankin \\ Division of Engineering, Brown University, Providence, Rhode Island 02912
}

Barbara L. Walden

Physics Department, Trinity College, Hartford, Connecticut 06106

Laura Riester

Oak Ridge National Laboratory, Oak Ridge, Tennessee 37831

(Received 29 December 2003; accepted 6 June 2004)

\begin{abstract}
Intrinsic tensile stresses in polycrystalline films are often attributed to the coalescence of neighboring grains during the early stages of film growth, where the energy decrease associated with converting two free surfaces into a grain boundary provides the driving force for creating tensile stress. Several recent models have analyzed this energy trade off to establish relationships between the stress and the surface/interfacial energy driving force, the elastic properties of the film, and the grain size. To investigate these predictions, experiments were conducted with diamond films produced by chemical vapor deposition. A multistep processing procedure was used to produce films with significant variations in the tensile stress, but with essentially identical grain sizes. The experimental results demonstrate that modest changes in the deposition chemistry can lead to significant changes in the resultant tensile stresses. Two general approaches were considered to reconcile this data with existing models of stress evolution. Geometric effects associated with the shape of the growing crystal were evaluated with a finite element model of stress evolution, and variations in the surface/interfacial energy driving force were assessed in terms of both chemical changes in the deposition atmosphere and differences in the crystal growth morphology. These attempts to explain the experimental results were only partially successful, which suggests that other factors probably affect intrinsic tensile stress evolution due to grain boundary formation. (c) 2004 American Institute of Physics. [DOI: 10.1063/1.1777811]
\end{abstract}

\section{INTRODUCTION}

Intrinsic tensile stresses that arise during the growth of polycrystalline films have long been associated with the coalescence of isolated grains into a continuous film. The free surfaces of neighboring grains usually have a higher total surface free energy than the grain boundary that forms when these two surfaces coalesce. This creates a driving force to pull the two surfaces together to form a new grain boundary segment, a process that can induce tensile stress in the adjacent islands. Hoffman first estimated the tensile stress associated with this phenomena as $M_{f}(\delta / 2 L)$, where $M_{f}$ is the biaxial modulus of the film, $L$ is the grain size, and $\delta$ is the gap between neighboring islands at the point where coalescence occurs. ${ }^{1,2}$ More recently, several researchers have analyzed the elastic energy that can develop when the surfaces of two adjacent islands first form a grain boundary. These results follow the general form proposed by Freund and Chason, ${ }^{3}$

$$
\frac{\sigma_{V}}{E}=A\left[\frac{\Delta \gamma}{E L}\right]^{B}
$$

where $\sigma_{V}$ is the average stress in the film, $E$ is the elastic modulus, and $L$ is the grain size. The energy difference that drives stress evolution is $\Delta \gamma=2 \gamma_{S}-\gamma_{B}$, where $\gamma_{S}$ is the free energy of the island surface and $\gamma_{B}$ is the free energy of the grain boundary that forms between neighboring islands. Freund and Chason analyzed different geometries that illustrate how the constants $A$ and $B$ can depend on the island shape and initial contact geometry. An earlier, simple energy balance with rectangular grains presented by Nijhawan et al. leads to an expression that obeys the form of Eq. (1), with a value of $B=0.5{ }^{4}$ Nix and Clemens also obtained this value for $B$ with a more sophisticated analysis of grain boundary formation for the contact between two cusped surfaces. ${ }^{5}$

The form of Eq. (1) predicts that the intrinsic tensile stresses that develop in a given material will depend only on geometric effects that determine the values of $A$ and $B$, along with the grain size and the energetic driving force due to $\Delta \gamma$ (i.e., for a film of a given material, with fixed elastic properties). The primary objective of the research reported here is to test these predictions with experiments where $\sigma_{V}$ varies while the grain size is held constant. These tensile stresses occur in a wide variety of different materials; ${ }^{6-9}$ however, in most cases this mechanism competes with compressive stresses that become dominant after individual islands coalesce into a dense film. Previous work shows that diamond formed by chemical vapor deposition (CVD) is an excellent system for conducting detailed studies of the tensile mechanism because significant compressive stresses do not mask the large tensile stresses. ${ }^{10}$ Sections II and III describe experimental observations in diamond films with a fixed grain 
TABLE I. Multistep process conditions for films grown at $800{ }^{\circ} \mathrm{C}$.

\begin{tabular}{llll}
\hline \hline Step & \multicolumn{1}{c}{ Duration } & \multicolumn{1}{c}{ Deposition conditions } & \multicolumn{1}{c}{ Purpose } \\
\hline I & $\begin{array}{l}1.5 \mathrm{~h}, \\
\text { longer in some cases }\end{array}$ & $\begin{array}{l}\left.1 \% \mathrm{CH}_{4} \text { (i.e., } C_{\mathrm{I}}=1 \%\right) \\
P=0.05 \text { atm, } 1100 \mathrm{~W}\end{array}$ & $\begin{array}{l}\text { Form basic microstructure under } \\
\text { identical conditions for all films } \\
\text { (i.e., same grain size, etc.) }\end{array}$ \\
II & $\begin{array}{l}1 \mathrm{hr} \text { when } C_{\mathrm{II}}=C_{\mathrm{I}}, \\
\text { variable for other } C_{\mathrm{II}} \\
\text { (see text) }\end{array}$ & $\begin{array}{l}\text { Same as step I, except one } \\
\text { condition is varied (i.e., } C_{\mathrm{II}} \\
\text { was varied in Figs. 3 and 4). }\end{array}$ & $\begin{array}{l}\text { Vary conditions after islands } \\
\text { coalesce }\end{array}$ \\
III & $\begin{array}{l}\text { Usually } 7.5 \mathrm{~h} \text { (total } \\
\text { time for steps I and III } \\
\text { is always } 9 \mathrm{~h} \text { ). }\end{array}$ & Same as step I & $\begin{array}{l}\text { Complete growth of a } 2.5 \mu \mathrm{m} \\
\text { thick film }\end{array}$ \\
\hline \hline
\end{tabular}

size, but with significant variations in the intrinsic stress. Possible explanations for these stress differences are considered in Sec. IV. The finite element model (FEM) in Sec. IV A is used to examine the validity of Eq. (1) as an approximate description of tensile stress during postcoalescence growth. This is necessary because the previous theoretical treatments associated with Eq. (1) evaluate stress only at the point where neighboring grains first impinge on each other, in contrast to our previous experiments with CVD diamond which demonstrate that most of the tensile stress develops after initial island coalescence is complete. ${ }^{10}$ In addition to the geometric effects that are analyzed with the FEM, Sec. IV B considers variations in $\Delta \gamma$ that can lead to changes in $\sigma_{V}$ [i.e., in accordance with Eq. (1)].

\section{EXPERIMENTAL APPROACH}

\section{A. Film growth}

Diamond was deposited onto 100 oriented Si substrates in a microwave-plasma CVD reactor, from $\mathrm{H}_{2} / \mathrm{CH}_{4}$ mixtures. The general procedures that were used are described in more detail elsewhere. ${ }^{10-12}$. Each set of experiments was conducted at substrate temperatures of either 800 or $900^{\circ} \mathrm{C}$. Variations in the $\mathrm{CH}_{4}$ concentration were used as the principle method for varying the stress, with all other conditions fixed (i.e., total pressure, input microwave power, and total gas flow). All samples were prepared using the same procedure to enhance nucleation via substrate abrasion with diamond powder.

Films with different stress levels and a fixed grain size were obtained by using the three-stage procedure outlined in Table I. For each set of samples, growth was initiated under identical conditions in stage I. For example, films deposited at $800^{\circ} \mathrm{C}$ were all grown for an initial period of $1.5 \mathrm{~h}$ with $1 \% \mathrm{CH}_{4}$. Based on previous work with these conditions, $1.5 \mathrm{~h}$ is approximately the point where the initial islands that form on the substrate coalesce into a continuous film. ${ }^{10}$ The growth conditions were varied during stage II only. During stage III, all films were grown under conditions that were identical to those during stage I (except for the time). One complication in this approach is that the growth rate changes when the $\mathrm{CH}_{4}$ concentration is varied. Thus, to produce similar film thicknesses for all samples during stage II, the growth time was also varied according to $t_{\mathrm{II}}=\left[C^{\circ} / C_{\mathrm{II}}\right]^{1 / 2}$, where $C_{\mathrm{II}}$ is the $\mathrm{CH}_{4}$ concentration during stage $\mathrm{II}$, and $C^{\circ}$ is the value of $C_{\mathrm{II}}$ for the control case (i.e., $1 \%$ for the $800^{\circ} \mathrm{C}$ data set and $0.4 \%$ for the $900^{\circ} \mathrm{C}$ data set). The exponent of $1 / 2$ that is used in this expression for varying $t_{\mathrm{II}}$ is an approximation based on the measured relationship between the growth rate and $C_{\mathrm{II}}$ for this particular range of conditions.

The multistep procedure in Table I allowed us to vary the intrinsic stress without significantly altering the grain structure of the films. Nucleation, growth, and coalescence of individual islands during stage I controls the grain structure of the film. While the microstructure continues to evolve after stage I, process variations during stage II do not alter the grain structure significantly because renucleation on existing grains is minimal. The use of stage III appears to complicate the growth procedure, however, it was employed to permit the growth of each film in a single day. This was necessary because resuming growth on subsequent days can lead to unpredictable variations in the experimental results. Previous experiments under similar conditions show that stage III corresponds to growth with a steady-state tensile stress $^{10}$ (i.e., stress-thickness vs time data with a constant slope, which implies that new material grows with a constant stress). In general, the intrinsic stress at the end of stage I was small (i.e., $<100 \mathrm{MPa}$ ). Almost all of the stress evolves during stages II and III, with most of the increase in stress occurring during stage II.

\section{B. Characterization}

The bending plate method was used to determine the total internal stress in the films. Radii of curvature were measured with a multibeam optical stress sensor. This apparatus is described elsewhere. ${ }^{13}$ The conventional procedure for analyzing curvature data relies on the assumption that the film has a uniform thickness, and that the film and substrate can only deform elastically. This leads to the following modified form of the Stoney equation: ${ }^{14}$

$$
\sigma_{V}=\frac{M_{S} H_{S}^{2}}{6 R H_{f}}\left[1+4 \frac{M_{f} H_{f}}{M_{S} H_{S}}-\frac{H_{f}}{H_{S}}\right] .
$$

At the deposition temperatures used here, the Si substrates can undergo some inelastic deformation. Our experimental investigations into the magnitude of this effect show that the diamond films grown at $800^{\circ} \mathrm{C}$ in the present study exhibit actual stress levels that are $5-15 \%$ lower than the values obtained with Eq. (2). ${ }^{15}$ In spite of this error, all of the stress 


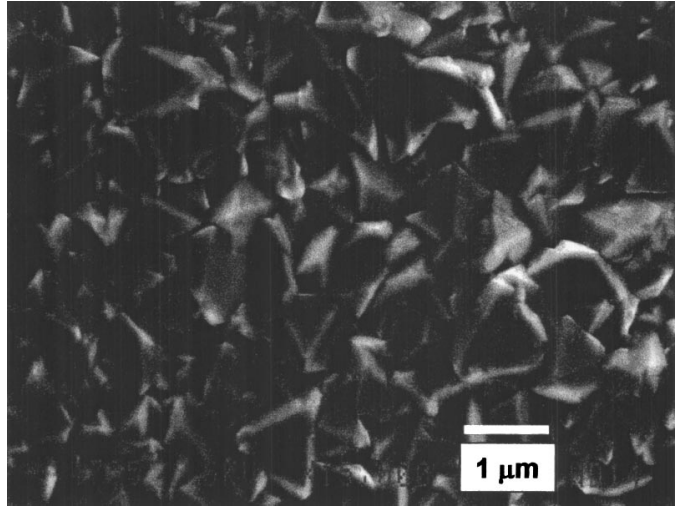

FIG. 1. SEM image of film grown at $800^{\circ} \mathrm{C}$ and $C_{\text {II }}=C_{\mathrm{I}}$ for 4 h (i.e., early in stage III).

values reported in Sec. III are based on Eq. (2), since a laborious set of measurements is needed to accurately correct the stress for each film. ${ }^{12}$ The error associated with inelastic substrate deformation can be reduced or eliminated by using lower deposition temperatures, however, the nondiamond content of these films increases at lower temperatures. Thus, growth temperatures below $800^{\circ} \mathrm{C}$ were not investigated here.

Information about the nondiamond content of the films was obtained with Raman spectroscopy and electron energy loss spectroscopy (EELS), with instrumentation and methods that we have described previously. ${ }^{11,16}$ Scanning electron microscopy (SEM) was used to determine film thickness and to view the grain structure. An example of the faceted top surface of one of these films is shown in Fig. 1. The conclusion that processing variations during stage II did not alter the grain structure or the final film thickness is based on these SEM observations. Transmission electron microscopy (TEM) was used to provide information about the grain boundary structure of selected samples.

The elastic modulus was determined by nanoindenting film cross sections. These specimens were prepared by cleaving samples from the backside of the Si substrate and then polishing the cross sections. Measurements were made with a Nanoindenter ${ }^{\mathrm{TM}}$ using a Berkovich diamond tip. The position of the indenter relative to the test surface of the specimen was constantly measured with a sensitive capacitance gauge. Plastic and elastic components of displacement are separated by continuous sensing of sample stiffness while force is being applied or held constant. Positions of the nanoindents on the film could not be verified microscopically because the response of the material was largely elastic as illustrated in Fig. 2. However, the distance from the microscope to the indenter was calibrated and indents were carefully selected to ensure a high probability of being positioned in the center of the film. Indentation on a smooth diamond surface should produce load vs displacement data with little or no plastic deformation and no change in deformation when load was held constant. Only data from these indents were considered for calculating the elastic modulus since load/displacement curves which indicated plastic deformation or displacement without corresponding increase in load were probably caused by indenter contact with surface as-

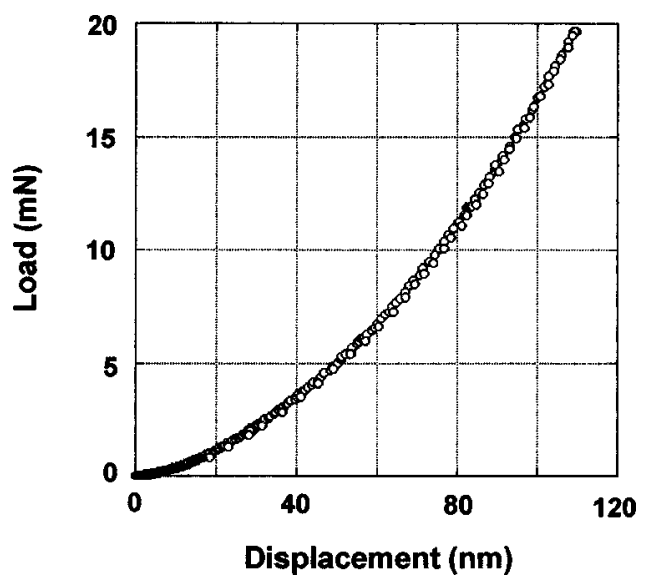

FIG. 2. Load vs displacement data for an indentation cycle, during the nanoindentation of a diamond film.

perities or contact on the edge rather than the center of the diamond film. The elastic modulus was determined by monitoring the contact stiffness during multiple loading segments, using the following relationship: ${ }^{17}$

$$
S=\frac{2 \beta}{\sqrt{\pi}} \sqrt{A} E_{r}
$$

where $A$ is the contact area, $E_{r}$ the reduced modulus, and $\beta$ an indenter geometry dependent constant (1.034 for the Berkovich indenter). The elastic modulus of the film material was extracted from $E_{r}$ using the following relationship:

$$
\frac{1}{E_{r}}=\frac{1-\nu^{2}}{E}+\frac{1-\nu_{o}^{2}}{E_{o}}
$$

where $E$ and $E_{0}$ are the Young's Modulus of the sample and the indenter, $\nu$ and $\nu_{0}$ the Poisson's ratio of the test material and the indenter, respectively. For each data point the stiffness was recorded, thus permitting calculation of the elastic properties over a wide range of penetration depths. If the contact stiffness varied a great deal as a function of penetration depth, it was assumed that the indenter made contact with an edge or an asperity and the data was discarded.

\section{RESULTS}

\section{A. Intrinsic stress}

The key variable in these experiments is the $\mathrm{CH}_{4}$ concentrations during stage II, which is denoted here as $C_{\mathrm{II}}$. Final stress measurements for sets of films grown at 800 and $900^{\circ} \mathrm{C}$ are shown in Figs. 3 and 4, respectively. Both figures exhibit the same general trend, with a maximum stress at intermediate $C_{\mathrm{II}}$, and significantly lower stresses at higher and lower values. The data reported in Figs. 3 and 4 were corrected for the compressive thermal stress that occurs during postdeposition cooling, $0.44 \mathrm{GPa}$ and $0.47 \mathrm{GPa}$ for 800 and $900{ }^{\circ} \mathrm{C}$ depositions, respectively. ${ }^{11}$ As noted in Sec. II, the stresses determined with Eq. (2) are based on the assumption that the substrate and film are fully elastic. For the samples grown at $800^{\circ} \mathrm{C}$, the error due to inelastic substrate deformation does not significantly alter any of our analysis or conclusions. Although these errors are larger for the 


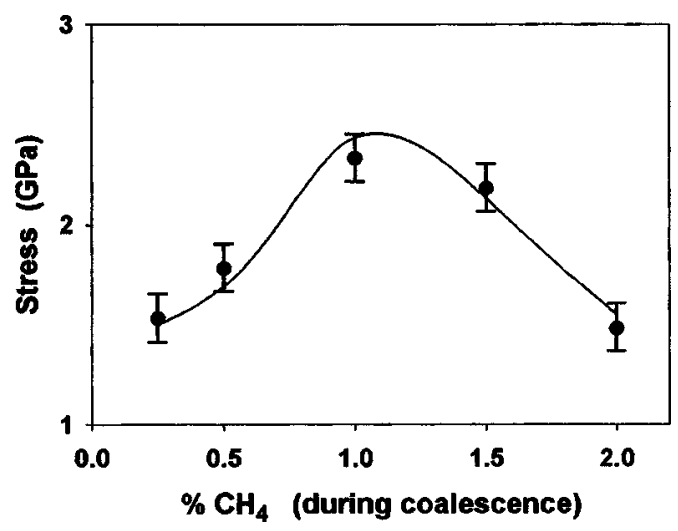

FIG. 3. Intrinsic stress values for films grown at $800^{\circ} \mathrm{C}$, as a function of the methane concentration during state II $\left(C_{\mathrm{II}}\right)$. All other conditions were identical for all films, as shown in Table I. Stresses were measured at room temperature. Reported values were corrected for $-0.44 \mathrm{GPa}$, to account for the compressive thermal stress induced upon cooling.

$900^{\circ} \mathrm{C}$ data, the intrinsic stress in these films still follows the trend seen in Fig. 4, such that these results apparently demonstrate the same basic phenomena at this higher temperature.

To investigate the effect of varying the temporal position of stage II, a set of experiments was conducted at $800^{\circ} \mathrm{C}$ with $C_{\mathrm{II}}=0.25 \%$. In this data set, three specimens were grown with stage I times of $1,2.5$, and $4 \mathrm{~h}$, such that stage II was started at a different time than the corresponding data point in Fig. 3 (i.e., where stage I was $1.5 \mathrm{~h}$ ). In all of these cases, the duration of stage II was still $2 \mathrm{~h}$ (i.e., identical to the results in Fig. 3). To obtain films with the same final thickness, stage III was altered to compensate for the difference in stage I. In other words, the total combined time for stage I and stage III was fixed at $9 \mathrm{~h}$ (e.g., when stage I was extended to $4 \mathrm{~h}$, stage III was reduced to $5 \mathrm{~h}$ ). These variations in the onset of stage II did not produce a discernable difference in the final stress state (i.e., the final values were all within $0.1 \mathrm{GPa}$ of one another, and $\sim 30 \%$ lower than the stress in the control experiment with $C_{\mathrm{II}}=1 \%$ ). Several specimens were also grown with stage II extended to longer times. These generally produced stresses that were only

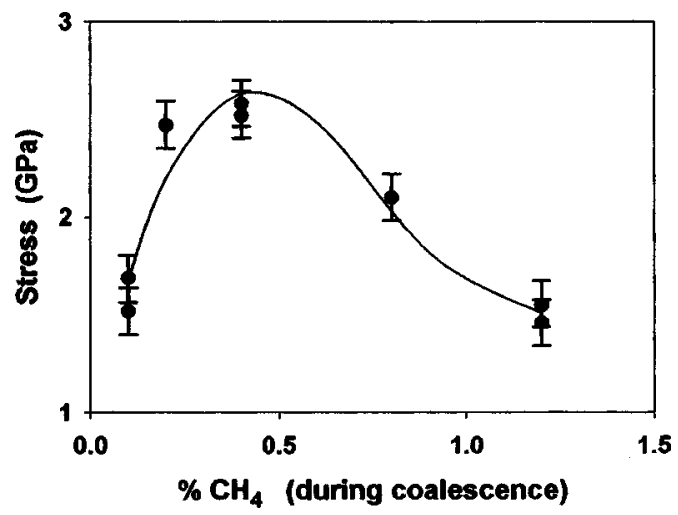

FIG. 4. Intrinsic stress values for films grown at $900^{\circ} \mathrm{C}$, as a function of $C_{\mathrm{II}}$. All other conditions were identical for all films, similar to Table I except that $C_{\mathrm{I}}=C_{\mathrm{III}}=0.4 \%$. Stresses were measured at room temperature. Reported values were corrected for $-0.47 \mathrm{GPa}$, to account for the compressive thermal stress induced upon cooling.

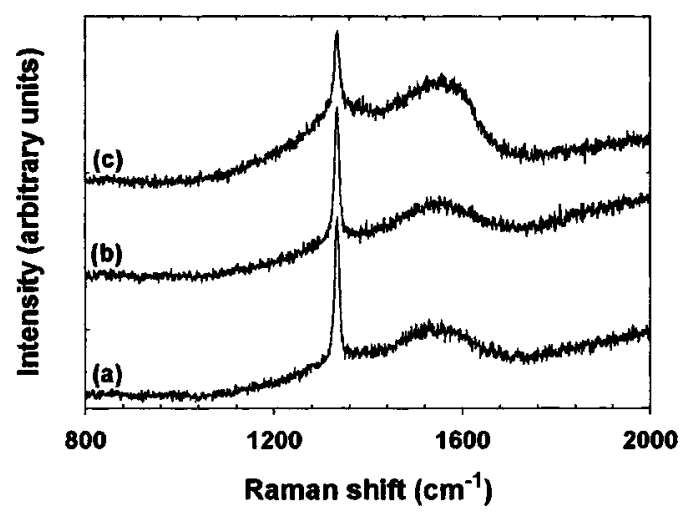

FIG. 5. Raman spectra for selected samples from Fig. 3. (a) $C_{\mathrm{II}}=0.1 \%$, (b) $C_{\mathrm{II}}=0.4 \%$, (c) $C_{\mathrm{II}}=1.2 \%$.

0.1-0.3 GPa lower, and the same basic trends seen in Fig. 3 were always observed (i.e., values of $C_{\mathrm{II}}$ above and below $1 \%$ produced lower final stresses).

The substantial variations seen in Figs. 3 and 4 led to several other preliminary investigations of the relationship between stress and processing chemistry. These were conducted at $800^{\circ} \mathrm{C}$, with the same basic methodology shown in Table I, except that the $\mathrm{CH}_{4}$ concentration was unchanged (i.e., $C_{\mathrm{II}}=1 \%$ ), and instead either the microwave power was varied or $\mathrm{O}_{2}$ was added to the inlet gas mixture during stage II. The conditions during stages I and III were the same as those used for the data set in Fig. 3. These changes in the stage II chemistry also produced noticeable variations in the final measured stress. The stress decreased with increasing microwave power. Specifically, decreasing the microwave power to $1100 \mathrm{~W}$ increased the stress by $14 \%$, while increasing the microwave power to $1250 \mathrm{~W}$ decreased the stress by $9 \%$. Stress decreases were also observed when small amounts of oxygen were added to the inlet gas, with a maximum decrease of $9 \%$ observed with an addition of $0.5 \%$. While these trends are interesting, the stress changes are smaller than those in Figs. 3 and 4. Thus, detailed characterization and analysis were focused on the films produced with $\mathrm{CH}_{4}$ variations.

\section{B. Nondiamond carbon and grain boundary structure}

Raman spectra from several of the films in Fig. 4 are shown in Fig. 5. The characteristic, strong diamond line at $1332 \mathrm{~cm}^{-1}$ is similar in all three cases. The shoulder at $1345 \mathrm{~cm}^{-1}$ and the broad peak centered near $1530 \mathrm{~cm}^{-1}$ reflect nondiamond carbon. These are similar for $C_{\mathrm{II}}$ values of $0.1 \%$ and $0.4 \%$, however, the spectrum for the film with $C_{\mathrm{II}}$ of $1.2 \%$ indicates that more nondiamond carbon is present. Similar Raman results were obtained for the films in Fig. 3 (i.e., differences in nondiamond carbon were not observed in films grown with $C_{\mathrm{II}}$ values of $0.5 \%$ and $1 \%$, while higher nondiamond carbon content was detected with $C_{\mathrm{II}}$ of $\left.2 \%\right){ }^{12}$ An increase in the nondiamond carbon content at higher $\mathrm{CH}_{4}$ concentrations is consistent with the findings of other researchers. ${ }^{18}$

Additional insight into the presence of nondiamond carbon was obtained with TEM. A distinct grain boundary phase was not observed in these materials. This is evident in the 
(a)

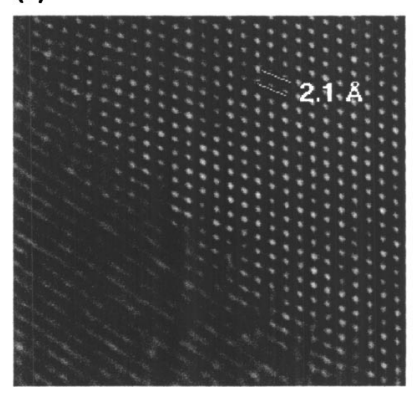

(b)

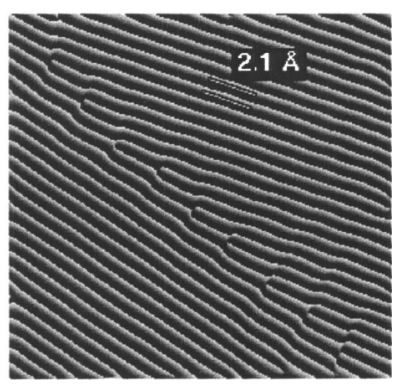

FIG. 6. (a) High resolution TEM image of grain boundary in material grown at $800^{\circ} \mathrm{C}$ and $C_{\mathrm{II}}=1 \%$. (b) Moire image obtained from Fig. 5(a).

high resolution electron microscopy (HREM) image in Fig. 6(a). The Moire image in Fig. 6(b) shows the position of grain boundary dislocations more clearly. Also, the bending of the Moire lines in Fig. 6(b) indicates that there are local strain variations near the grain boundary. A comparison between EELS data obtained from a bulk grain and a grain boundary is shown in Fig. 7. The band near $21-25 \mathrm{eV}$ indicates the nondiamond carbon content is enhanced near the grain boundary. ${ }^{16,19,20}$ Thus the combination of the HREM and the EELS observations indicates that nondiamond carbon does not form a distinct grain boundary phase, while there is some enhancement of nondiamond carbon in the vicinity of the grain boundary. This appears to apply to the entire range of $C_{\text {II }}$ values that was studied, although it is important to remember that TEM studies are inherently limited to very small portions of the film.

Previous work indicates that variations in the nondiamond carbon content of CVD diamond can alter the elastic modulus. For example, Savvides and Bell report diamond moduli of $500-533 \mathrm{GPa}$ in diamond and values of 62-213 GPa in diamondlike carbon. ${ }^{21}$ DeFazio et al. observed elastic moduli ranging from 413 to $941 \mathrm{GPa}$ in CVD diamond..$^{22}$ It is possible that some of the discrepancy in the literature reflects the inherent difficulty of making diamondtip indents on a diamond film (the films are typically somewhat softer than the tip, but both materials are extremely hard). Even with these difficulties, the general consensus in the literature is that the modulus decreases with increasing nondiamond carbon content. ${ }^{23}$ To evaluate this effect in our

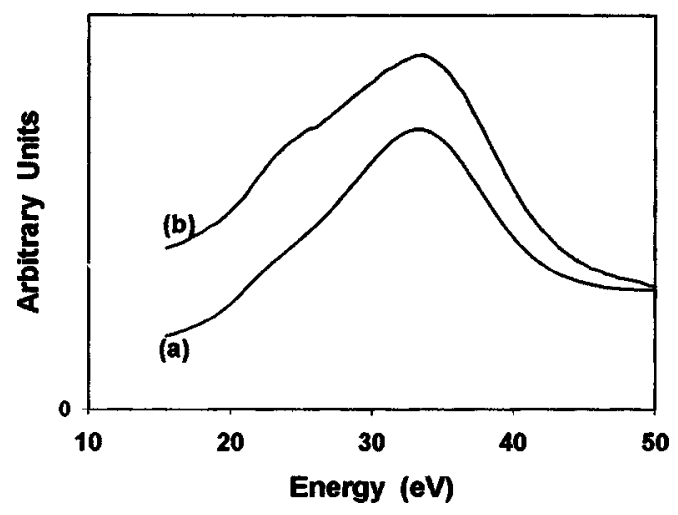

FIG. 7. Electron energy loss spectra obtained in the TEM, for material grown at $800^{\circ} \mathrm{C}$ and $C_{\mathrm{II}}=1 \%$. (a) Bulk diamond grain (far away from grain boundary), (b) probe located on grain boundary.

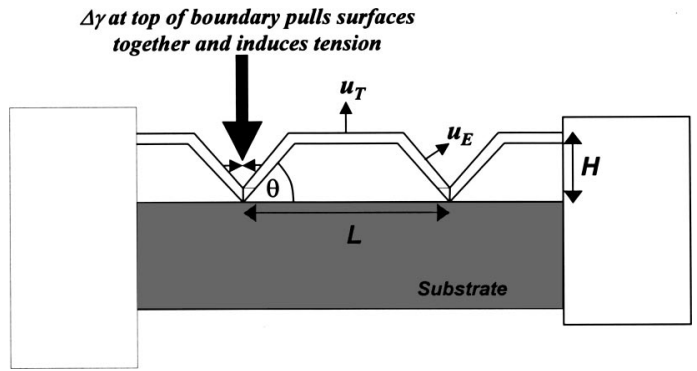

FIG. 8. Schematic of island growth and coalescence that is used for the FEM. The dotted line shows islands at the point where impingement first occurs.

films, nanoindentation was used to measure the elastic modulus of materials with different nondiamond carbon contents. For films grown at $800^{\circ} \mathrm{C}$ and $C_{\text {II }}$ values of $1 \%$ and $2 \%$, the biaxial moduli determined by nanoindentation were $971 \mathrm{GPa}$ and $800 \mathrm{GPa}$, respectively. This variation in $M_{f}$ with increasing $C_{\mathrm{II}}$ produces a relatively small difference in the stress value obtained from curvature measurements and Eq. (2) (i.e., it does not significantly alter the values in Figs. 3 and 4). However, the decrease in $M_{f}$ means that the lower stress measured with higher $C_{\mathrm{II}}$ does not correspond to an equivalent decrease in strain. While the impact of a lower value of $M_{f}$ is reflected in Eq. (1), the measured drop in $M_{f}$ is not the primary factor responsible for the lower observed stresses. To see this, note that the exponent $C$ in Eq. (1) is generally less than 1 , such that the measured modulus decrease of $17 \%$ corresponds to less than half of the observed stress decrease of $35 \%$ observed in Fig. 3.

\section{ANALYSIS}

\section{A. Finite element modeling}

As noted in Sec. I, several researchers have analyzed the stress generated by the initial contact between neighboring islands to obtain results that fit the form of Eq. (1), while our previous results with CVD diamond demonstrate that most of the stress evolves after initial island impingement. ${ }^{10} \mathrm{Be}-$ fore considering possible explanations for the measured stress variations in Figs. 3 and 4, we use FEM to assess whether Eq. (1) is a valid scaling law for intrinsic tensile stress evolution that occurs after islands initially impinge. This phenomenon, depicted in Fig. 8, was recently analyzed with a similar FEM approach. A more detailed description of this methodology is presented elsewhere. ${ }^{12,24}$ The principle concept employed in this model is that the energy trade off between $\Delta \gamma$ and elastic strain is now extended to grain boundary formation that occurs during film growth following initial island coalescence.

The FEM treats cases where the film thickness $H$ and the grain size $L$ are much smaller than the film width (i.e., the dimension into the page in Fig. 8). This leads to a planestrain type of calculation, similar to thin-film stress models that have been used elsewhere. ${ }^{5,14}$ Freund refers to this as a two dimensional contact. ${ }^{3}$ In this classification system, a three dimensional contact emanates from the point contact between two spheres (or between truncated spheres). While configurations of this type are likely to be common during 
initial coalescence, they are not prevalent in the growth of a continuous film. Here most of the cusps between neighboring grains will more closely resemble a two-dimensional contact, although the actual stress distributions will certainly be more complex than those obtained from the FEM. Following our previous treatment, uniform islands begin to grow, initially separated from their neighbors by a uniform distance $L$ (i.e., the grain size). Each island consists of two types of facet, a top facet and two edge facets. When neighboring islands impinge, the edge facets of the neighboring grains form a notch over the grain boundary. As film growth proceeds, the grain boundary becomes longer until the notch eventually disappears when the film thickness reaches

$$
H^{*}=\frac{L \sin \theta}{2\left[\frac{u_{E}}{u_{T}}-\cos \theta\right]},
$$

where $\theta$ is the angle that defines the edge facet orientation (see Fig. 8), and the growth rates of the top and edge facets are $u_{T}$ and $u_{E}$, respectively. At this point, all of the edge facets in the film disappear, leaving a flat surface comprising the top facet of each grain. When $H^{*}$ is reached the tensile stress stops increasing, and further deposition produces stress associated with templated growth onto the already strained top surface of the film. This essentially assumes that there are no operative mechanisms for stress relaxation, such that subsequent atomic layers that are deposited will adopt the lattice positions and hence the strain of the underlying film. This behavior is similar to the steady-state stress observed experimentally (described at the end of Sec. III A).

The FEM calculations describe the complete stress distribution throughout the film, however, comparison with the form of Eq. (1) requires a scalar value. This was taken as the average steady-state stress $\sigma^{*}$ associated with the deposition of an incremental layer on top of the planar film. To obtain this value, the FEM was run to the point where the edge facets (i.e., the notch) first disappears at film thickness $H^{*}$, and $\sigma^{*}$ was obtained as the average in-plane stress in the top layer of the film. This differs from $\sigma_{V}$ in Eq. (1), which is defined as an average value over the entire film volume. However, the value of $\sigma^{*}$ should approach $\sigma_{V}$ when the film thickness is much greater than $H^{*}$ (i.e., because $\sigma^{*}$ corresponds to the average stress for material deposited beyond $H^{*}$, in the absence of other stress generation or relaxation mechanisms).

The results in Fig. 9 show the effect of individually varying $\Delta \gamma, L$, and $M_{f}$ [i.e., the three physical quantities on the right-hand side of Eq. (9)]. The island geometry was fixed for all of these calculations, with $\theta=45^{\circ}$ and $u_{T} / u_{E}=0.5$. Fitting the results in Figs. 9(a)-9(c) to the form of Eq. (1) gives an average value of $B=0.53$. While this value is close to the Nijhawan-Nix-Clemens exponent of 0.5 , this type of fit should be understood as an approximate scaling law and not an exact result. Also, note that individually fitting each of the data sets in Fig. 9 gives slightly different values [e.g., varying only $\Delta \gamma$ in Fig. 9(a) gives $B=0.54$ ]. Following the logic that leads to Eq. (5), the values of $\theta$ and $u_{T} / u_{E}$ will dictate the geometric evolution of the film. The FEM shows
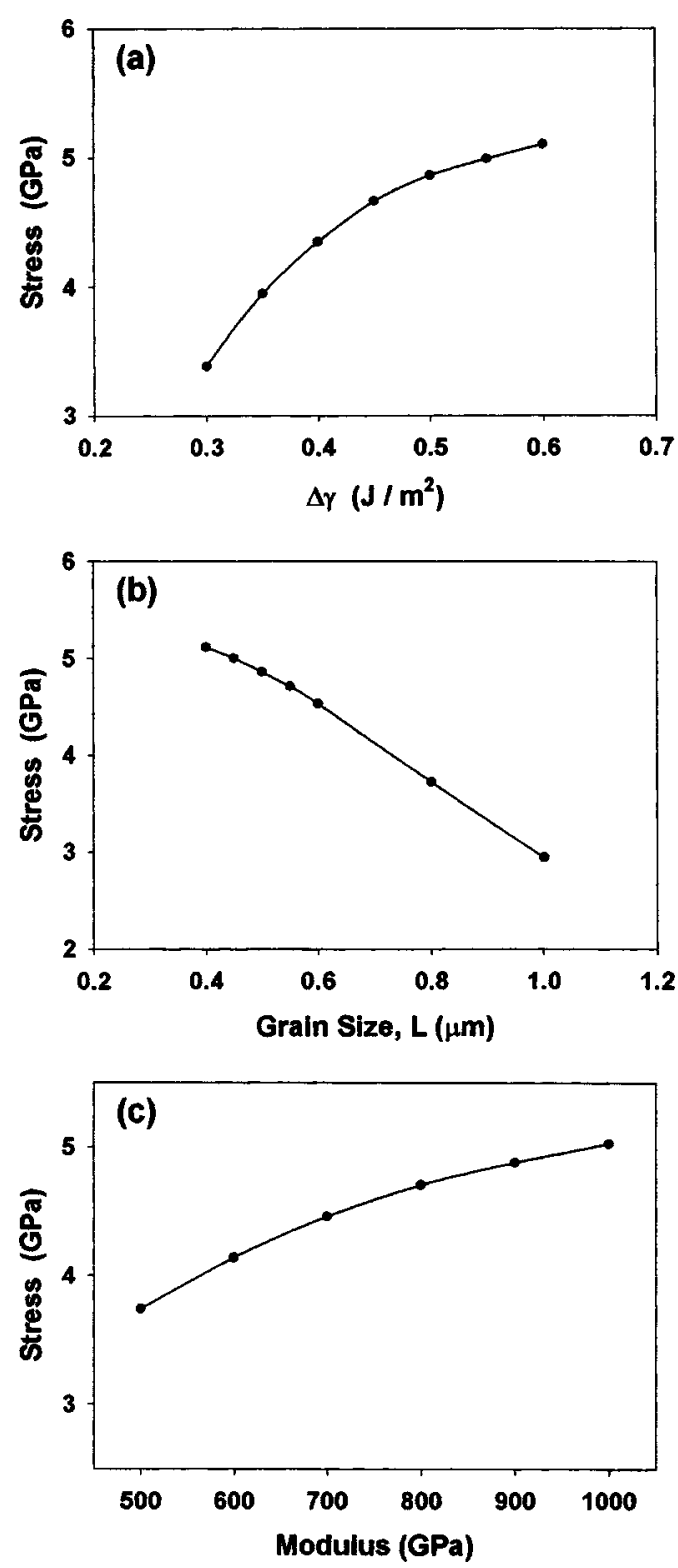

FIG. 9. FEM results for $\theta=45^{\circ}$ and $u_{T} / u_{E}=0.5$ : (a) $L=0.5 \mu \mathrm{m}, M_{f}$ $=900 \mathrm{GPa}$, and $\Delta \gamma$ varied as shown. (b) $M_{f}=900 \mathrm{GPa}, \Delta \gamma=0.5 \mathrm{~J} / \mathrm{m}^{2}$, and $L$ varied as shown. (c) $L=0.5 \mu \mathrm{m}, \Delta \gamma=0.5 \mathrm{~J} / \mathrm{m}^{2}$, and $M_{f}$ varied as shown.

that varying these parameters changes the value of $B$. For example, the average value of $B$ increases to 0.61 when results analogous to those in Fig. 9 are obtained with $\theta=60^{\circ}$ and $u_{T} / u_{E}=1$.

For the cases that we have investigated, Eq. (1) provides a reasonable approximation of the FEM results for a given island geometry (i.e., for fixed values of $\theta$ and $u_{T} / u_{E}$ ). This suggests two possible explanations for the observed stress variations in Fig. 3 that are consistent with Eq. (1). One is that changing the $\mathrm{CH}_{4}$ concentration alters the so called island geometry and the other is that $\Delta \gamma$ varies. Geometric issues are considered here with further reference to the FEM calculations, and $\Delta \gamma$ is discussed in Sec. IV B.

It is well established that CVD diamond grows with two dominant facets, $\langle 111\rangle$ and $\langle 100\rangle .{ }^{25}$ This is more complex than the model island geometry in Fig. 8, however, the FEM still provides relevant insight into the general effects of 


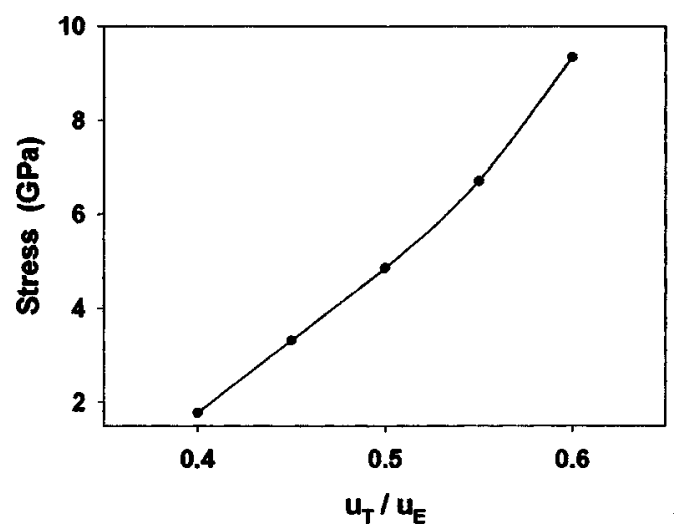

FIG. 10. FEM results showing the effect of varying $u_{T} / u_{E}\left(\theta=45^{\circ}, L\right.$ $=0.5 \mu \mathrm{m}, M_{f}=900 \mathrm{GPa}$, and $\Delta \gamma=0.5 \mathrm{~J} / \mathrm{m}^{2}$ ).

changing the crystal geometry. With microwave-plasma CVD conditions similar to those used to obtain the data in Fig. 3, $\langle 111\rangle$ facets are dominant for $\mathrm{CH}_{4}$ concentrations below $0.4 \%$ and $\langle 100\rangle$ facets become increasingly prevalent as the $\mathrm{CH}_{4}$ concentration is increased from $0.4 \%$ to $1.2 \% .^{26-28}$. This transition implies that the $\langle 111\rangle$ growth rate increases faster than the $\langle 100\rangle$ growth rate. Interpreting this effect with the simpler two-dimensional FEM geometry corresponds to varying $u_{T} / u_{E}$ while $\theta$ is fixed (i.e., because varying $\theta$ would correspond to a different set of facets). A larger value of $u_{T} / u_{E}$ with fixed $\theta$ and $L$ increases $H^{*}$ according to Eq. (5) (i.e., the edge facet grows out of the film at a larger thickness). This effect leads to a larger stress, as seen in the FEM results in Fig. 10. Because the actual diamond grain morphology differs considerably from the assumed model geometry, it is not possible to determine whether the relative change in $\langle 100\rangle$ and $\langle 111\rangle$ growth rates will cause an increase or decrease in stress with increasing $\mathrm{CH}_{4}$. However, the FEM results in Fig. 10 suggest that this type of geometric change could contribute to and possibly dominate one of the two trends observed in Fig. 3 (i.e., the increasing stress from $0.25 \%$ to $1 \% \mathrm{CH}_{4}$, or the decreasing stress from $1 \%$ to $2 \%$ $\mathrm{CH}_{4}$ ).

As noted above, the FEM results demonstrate that Eq. (1) can serve as a reasonable scaling law to describe the maximum tensile stress during postcoalescence growth. This result is not necessarily obvious, since derivations leading to Eq. (1) have been based on the initial contact between two islands, rather than on the grain boundary formation mechanism depicted in Fig. 8. It is important to note that the microstructure evolution described by the FEM is oversimplified in a number of ways. First, the diamond films studied here have relatively rough surfaces instead of the eventual smooth surface that evolves at $H^{*}$ in the model. Also, the model geometry ignores a number of other factors such as grain boundary grooving and stress distributions caused by variations in grain sizes and grain positions. Evaluating these effects is potentially relevant, but beyond the scope of our current analysis. Several three-dimensional FEM calculations were also performed with an analogous island geometry (i.e., four edge facets instead of two). These results show stress evolution that is generally similar to the 2D model, in that the initial contact between islands produces a very small average stress in the film, such that most of the stress evolves after the initial contact event. However, these 3D calculations are significantly more time consuming and more detailed comparisons were not conducted.

\section{B. Surface and interface energies}

Another approach to interpreting our experimental results with Eq. (1) is to consider whether variations in $C_{\mathrm{II}}$ will produce chemical changes that alter the value of either $\Delta \gamma$ or $M_{f}$ (i.e., as opposed to the geometric changes discussed in the preceding section). As noted in Sec. III B, the stress decrease at higher $C_{\mathrm{II}}$ cannot be attributed solely to the measured decrease in $M_{f}$. To consider the possibility of large changes in $\Delta \gamma$, Eq. (1) can be rearranged to obtain $\Delta \gamma$ values that would correspond to the measured stress variations in Fig. 3 [i.e., assuming that Eq. (1) is valid]. In doing this, note that the value of $B$ has a large effect on the magnitude of $\Delta \gamma$ that is obtained from this type of calculation. With $B=0.5$ (Nijhawan-Nix-Clemens result) the known values for $\sigma_{V}, L$, and $M_{f}$ lead to $\Delta \gamma$ values of roughly $1-2 \mathrm{~J} / \mathrm{m}^{2}$, while $B$ $=2 / 3$ (Freund-Chason results for 2D contact) gives $150-300 \mathrm{~J} / \mathrm{m}^{2}$. The FEM results in the preceding section suggest $B$ values that are between these two limits, however, $\Delta \gamma$ values on the order of several hundreds of $\mathrm{J} / \mathrm{m}^{2}$ are physically unrealistic. Diamond has relatively large surface and grain boundary energies, such that a value of $\Delta \gamma$ on the order of $1 \mathrm{~J} / \mathrm{m}^{2}$ is reasonable, thus we first use $B=0.5$ to evaluate the measured stress variations in Fig. 3. This indicates that the stress increase going from $C_{\mathrm{II}}$ of $0.25 \%$ to $1 \%$, corresponds to a $\Delta \gamma$ increase of $130 \%$, while for the measured stress decline going to $C_{\mathrm{II}}=2 \%, \Delta \gamma$ must decrease by $51 \%$ (this latter value is adjusted for the measured modulus decrease described in Sec. III). Essentially the same conclusion is reached with different values of $B$. For example, performing similar calculations with the unrealistic $\Delta \gamma$ values obtained with $B=2 / 3$ also leads to large variations in the predicted $\Delta \gamma$ (an $87 \%$ increase in $\Delta \gamma$ in going from $C_{\mathrm{II}}$ of $0.25 \%$ to $1 \%$, and a $32 \%$ decrease in $\Delta \gamma$ going from $C_{\mathrm{II}}$ of $1 \%$ to $2 \%$ ).

If changes in the deposition chemistry alter $\Delta \gamma$, this must correspond to changes in the grain boundary energy $\gamma_{b}$, and/or the surface energy $\gamma_{S}$. While the HREM results do not show evidence of a distinct graphitic phase, it is possible that the interfacial energy $\gamma_{b}$ is altered by local graphitic bonding that is suggested by the EELS results in Fig. 7. If variations in $\gamma_{b}$ were to account for the lower $\sigma_{V}$ (Figs. 3 and 4) and more nondiamond carbon (Fig. 5), then an increase in graphitic bonding at the grain boundaries would have to produce a significant increase in the interfacial energy. Atomistic modeling has been used to investigate the role of $\pi$ bonding (i.e., graphitic carbon) at diamond grain boundaries. ${ }^{29,30}$ These calculations indicate that different interface configurations involving graphitic bonding can either increase or decrease $\gamma_{b}$. However, the magnitude of these variations does not appear to be large enough to account for the observed stress decreases in Figs. 3 and 4 [i.e., by altering the value of $\Delta \gamma$ in Eq. (1)]. 
Large variations in $\gamma_{S}$ for a given type of facet are most likely to be associated with surface hydrogenation. This assertion is based on detailed analysis and experimental data indicating that deposition under standard conditions $\left(\sim 1 \% \mathrm{CH}_{4}\right)$ will lead to surfaces that are roughly $85 \%$ hydrogenated. $^{31-33}$ These studies indicate that the primary reactions associated with surface hydrogen are

$$
\begin{aligned}
& C_{d}^{*}+\mathrm{H} \Leftrightarrow C_{d} \mathrm{H}, \\
& C_{d} \mathrm{H}+\mathrm{H} \Leftrightarrow C_{d}^{*}+\mathrm{H}_{2},
\end{aligned}
$$

where $C_{d} \mathrm{H}$ and $C_{d}^{*}$ denote hydrogenated surface sites and unoccupied surface radical sites, respectively. Growth occurs via carbon adsorbates that incorporate at $C_{d}^{*}$ sites, however, the concentration of these adsorbates is believed to be relatively small. ${ }^{31,32}$ Thus, the growth surface primarily comprises $C_{d} \mathrm{H}$ and $C_{d}^{*}$ (i.e., hydrogentated sites and dangling bonds). We first apply this knowledge of the surface chemistry to assess our experimental observation of decreasing stress with increasing microwave power. Lang et al. used actinometry to show that the atomic hydrogen in the plasma increases at higher microwave power. ${ }^{34}$ Experiments show that this increase in atomic hydrogen decreases the surface hydrogenation. ${ }^{35}$ In general, $\gamma_{S}$ should increase with decreasing $C_{d} \mathrm{H}$. This would then increase $\Delta \gamma$, which suggests a higher stress via Eq. (1), rather than the lower stress that was observed.

Experiments and models also indicate that increasing the methane concentration produces a small decrease in the atomic hydrogen concentration. ${ }^{36,37}$ Following the surface hydrogenation discussion above, this should cause a small decrease in $\gamma_{S}$, whereby Eq. (1) predicts a small decrease in stress. While this could influence the observed stress variations, this contribution is unlikely to be large enough to fully explain any of the results in Figs. 3 and 4. For example, a simple approach is to consider bond energy contributions for $C_{d} \mathrm{H}\left[-5.59(10)^{-20} \mathrm{~J}\right]$ and $C_{d}^{*}\left[2.87(10)^{-19} \mathrm{~J}\right] .^{38}$ While the precise quantitative accuracy of this approach is dubious, these values suggest that a $2 \%$ change in surface hydrogenation corresponds to $\gamma_{S}$ variations on the order of $\sim 0.1 \mathrm{~J} / \mathrm{m}^{2}$. This would correspond to stress variations of less than $0.1 \mathrm{GPa}$ according to Eq. (1). Increasing $\mathrm{CH}_{4}$ is expected to produce less than a $2 \%$ change in surface hydrogenation, ${ }^{36,37}$ so this does not appear to have a dominant influence on the results in Figs. 3 and 4.

While the surface hydrogenation effects considered above do not appear to be consistent with the experimentally measured stress variations, changes in the dominant facets could produce significant changes in $\Delta \gamma$. As noted in Sec. IV A, increasing the methane concentration is known to shift the growth morphology from $\langle 111\rangle$ to $\langle 100\rangle$ facets. ${ }^{25-27} \mathrm{~A} \Delta \gamma$ increase or decrease on the order of $\sim 1 \mathrm{~J} / \mathrm{m}^{2}$ could be caused by the difference in $\gamma_{S}$ for these facets or by changes in $\gamma_{b}$ associated with different grain boundary structures (i.e., due to the change in facet morphology). These types of changes are thus large enough to explain the magnitude of some of the stress variations in Figs. 3 and 4.

\section{DISCUSSION AND CONCLUSIONS}

The data in Sec. III show, somewhat surprisingly, that relatively modest changes in the deposition chemistry can produce significant variations in the intrinsic stress. While the scaling law in Eq. (1) can provide a reasonable approximation of the more detailed computational results reported in Sec. IV A, attempts to interpret the experimental results with Eq. (1) were only partially successful. As noted in Sec. IV B, possible changes in $\gamma_{b}$ and $\gamma_{S}$ associated with nondiamond carbon and surface hydrogenation appear to be too small to explain the magnitude of the observed stress variations. The expected change in facet morphology with increasing methane concentrations could, in theory, lead to larger stress variations due to geometric effects (Sec. IV A) and/or differences in $\Delta \gamma$ associated with the different facets. However, this change in faceting does not appear to be consistent with the observation that the stresses in Figs. 3 and 4 go through a maximum with increasing $C_{\mathrm{II}}$. Changes in the facet morphology also fail to explain the decrease in stress as the microwave power is increased.

Since our current understanding of diamond film growth and stress evolution does not fully account for the experimental observations, additional considerations are needed. The limitations encountered in interpreting the data with Eq. (1) can be assessed either in terms of $\Delta \gamma$ and the exponent $B$, or as evidence that the energy analysis that leads to Eq. (1) does not fully describe tensile stress evolution. In trying to apply Eq. (1), it is possible that there are factors that have not been fully considered with our relatively simple approaches. For example, real film microstructures are more complex than the periodic island array used for the FEM, and it is always difficult to make quantitative assessments of surface and grain boundary energies. However, the tensile stress evolution in CVD diamond may also be affected by other mechanisms that are not considered in the energetic analysis that leads to Eq. (1). In particular, it is possible that the kinetics of both film growth and stress evolution are important in determining the intrinsic stress. This does not necessarily contradict the methodology associated with Eq. (1) and our FEM treatment. Instead, these energy based approaches should determine a limiting value that may or may not be reached because of certain kinetic factors. Consideration of these possibilities will require further study.

Although the analyses in Sec. IV do not provide a conclusive interpretation of the experimental results, the explanations that were explored demonstrate that applying Eq. (1) to real situations can be rather complicated, in spite of the relatively simple form of this expression. At first glance the form of Eq. (1) suggests that large intrinsic stress variations should not be observed in films with a fixed composition and a fixed grain size. However, the experimental results in Sec. III contradict this interpretation. The analyses in Sec. IV A suggest that variations in the surface topography of the film can have a significant effect on stress evolution (independent of grain size), and the considerations in Sec. IV B demonstrate possible relationships between $\Delta \gamma$ and the chemistry of the deposition environment. Beyond investigations of the validity of Eq. (1), the experimental results in Sec. III are also 
of interest because they show that variations in the deposition environment can be used to control and vary the intrinsic stress in CVD diamond. Further research on these effects can potentially lead to improved strategies for controlling stress levels and stress gradients during film growth.

\section{ACKNOWLEDGMENTS}

Primary research support from the National Science Foundation, under Award No. DMR-0305418 is gratefully acknowledged. This work also made use of MRSEC Shared Experimental Facilities, supported by NSF under Award No. DMR-0079964. We are also grateful to Professor Allan Bower of Brown University for assistance with the FEM.

${ }^{1}$ R. W. Hoffman, Phys. Thin Films 3, 211 (1966).

${ }^{2}$ F. A. Doljack and R. W. Hoffman, Thin Solid Films 12, 71 (1972).

${ }^{3}$ L. B. Freund and E. Chason, J. Appl. Phys. 89, 4866 (2001).

${ }^{4}$ S. Nijhawan, J. Rankin, B. L. Walden, and B. W. Sheldon, in Thin-FilmsStresses and Mechanical Properties VII, edited by R. C. Cammarata, E. P. Busso, M. Nastasi, and W. C. Oliver, Mater. Res. Soc. Symp. Proc. No. 505 (Materials Research Society, Pittsburgh, 1998) p. 415.

${ }^{5}$ W. D. Nix and B. M. Clemens, J. Mater. Res. 14, 3467 (1999).

${ }^{6}$ R. Abermann and R. Koch, Thin Solid Films 129, 71 (1985).

${ }^{7}$ R. Koch, J. Phys.: Condens. Matter 6, 9519 (1994).

${ }^{8}$ A. L. Shull and F. Spaepen, J. Appl. Phys. 80, 6243 (1996).

${ }^{9}$ J. Floro, S. J. Hearne, J. A. Hunter, P. Kotula, E. Chason, S. C. Seel, and C. V. Thompson, J. Appl. Phys. 89, 4886 (2001).

${ }^{10}$ B. W. Sheldon, A. Lau, and A. Rajamani, J. Appl. Phys. 90, 5097 (2001).

${ }^{11}$ S. Nijhawan, S. M. Jankovsky, B. W. Sheldon, and B. L. Walden, J. Mater. Res. 14, 1046 (1999).

${ }^{12}$ A. Rajamani, Ph.D Thesis, Brown University, 2003.

${ }^{13}$ E. Chason and B. W. Sheldon, Surf. Eng. 19, 387 (2003).

${ }^{14}$ L. B. Freund and S. Suresh, Thin Film Materials (Cambridge University Press, Cambridge, 2003).

${ }^{15}$ A. Rajamani, A. Bhandari, and B. W. Sheldon (unpublished).
${ }^{16}$ B. W. Sheldon, R. Csencsits, J. Rankin, R. E. Boekenhauer, and Y. Shigesato, J. Appl. Phys. 75, 5001 (1994).

${ }^{17}$ W. C. Oliver and G. M. Pharr, J. Mater. Res. 7, 1564 (1992).

${ }^{18}$ S. K. Choi, D. Y. Jung, and H. M. Choi, J. Vac. Sci. Technol. A 14, 165 (1996).

${ }^{19}$ Y. Wang, R. W. Hoffman, and J. C. Angus, J. Vac. Sci. Technol. A 8, 2226 (1990).

${ }^{20}$ D. N. Belton and S. J. Schmeig, J. Vac. Sci. Technol. A 8, 2353 (1990).

${ }^{21}$ N. Savivides and T. J. Bell, J. Appl. Phys. 72, 2791 (1992).

${ }^{22}$ L. DeFazio, S. Syngellakis, R. J.K. Wood, F. M. Fugiuele, and G. Sciume, Diamond Relat. Mater. 10, 765 (2001).

${ }^{23}$ H. Noguchi, Y. Kubota, and I. Okada, J. Vac. Sci. Technol. B 16, 2772 (1998).

${ }^{24}$ A. Rajamani, B. W. Sheldon, E. Chason, and A. F. Bower, Appl. Phys. Lett. 81, 1204 (2002).

${ }^{25}$ H. Liu and D. S. Dandy, Diamond Chemical Vapor Deposition (Noyes Publications, Park Ridge, N.J., 1995), Chap. 3.

${ }^{26}$ K. Kobashi, K. Nishimura, Y. Kawate, and T. Horiuchi, Phys. Rev. B 38, 4067 (1988).

${ }^{27}$ K. Kobashi, K. Nishimura, K. Miyata, K. Kumagai, and A. Nakaui, J. Mater. Res. 5, 2469 (1990).

${ }^{28}$ C. Wild, R. Kohl, N. Herres, W. Mullerschert, and P. Koidl, Diamond Relat. Mater. 3, 373 (1994).

${ }^{29}$ O. A. Shenderova and D. W. Brenner, in Defects in Electronic Materials II, edited by J. Michel, T. Kennedy, K. Wada, and K. Thake,Mater. Res. Soc. Symp. Proc. No. 442 (Materials Research Society, Pittsburgh, 1997), p. 693.

${ }^{30}$ O. A. Shenderova and D. W. Brenner, Phys. Rev. B 60, 7053 (1999).

${ }^{31}$ D. G. Goodwin, J. Appl. Phys. 74, 6888 (1993).

${ }^{32}$ M. Frenklach and S. Skokov, J. Phys. Chem. B 101, 3025 (1997).

${ }^{33}$ C. C. Battaile, D. J. Srolovitz, and J. E. Butler, J. Mater. Res. 14, 6293 (1999).

${ }^{34}$ T. J. Lang, J. Stiegler, Y. Von Kaenel, and E. Blank, Diamond Relat. Mater. 5, 1171 (1996).

${ }^{35}$ I. Sakaguchi, M. Nishitani-gamo, K. P. Loh, H. Haneda, and T. Ando, J. Appl. Phys. 86, 1306 (1999).

${ }^{36}$ W. L. Hsu, Appl. Phys. Lett. 59, 1427 (1991).

${ }^{37}$ D. S. Dandy and M. E. Coltrin, J. Appl. Phys. 76, 3102 (1994).

${ }^{38}$ Y. F. Zhang, F. Q. Zhang, and G. H. Chen, Mater. Lett. 20, 39 (1994). 